ALEA, Lat. Am. J. Probab. Math. Stat. 14, 327-336 (2017)

DOI: $10.30757 /$ ALEA.v14-18

\title{
Non-universality for longest increasing subsequence of a random walk
}

\author{
Robin Pemantle and Yuval Peres \\ Department of Mathematics, University of Pennsylvania \\ 209 S. 33rd Street, \\ Philadlephia, PA 19104, USA. \\ E-mail address: pemantle@math.upenn.edu \\ Microsoft Research \\ 1 Microsoft Way, \\ Redmond, WA 98052, USA. \\ E-mail address: peres@microsoft.com
}

\begin{abstract}
The longest increasing subsequence of a random walk with mean zero and finite variance is known to be of length $n^{1 / 2+o(1)}$. We show that this is not universal for symmetric random walks. In particular, the symmetric Ultra-fat tailed random walk has a longest increasing subsequence that is asymptotically at least $n^{0.690}$ and at most $n^{0.815}$. An exponent strictly greater than $1 / 2$ is also shown for the symmetric stable- $\alpha$ distribution when $\alpha$ is sufficiently small.
\end{abstract}

\section{Introduction}

It is well known that the longest increasing subsequence (LIS) of a sequence of $n$ IID non-atomic random variables has length $(2+o(1)) \sqrt{n}$ with high probability. (see Veršik and Kerov 1977; Logan and Shepp 1977). A different model was considered by Angel et al. (2012). Let $S_{n}:=\sum_{k=1}^{n} X_{k}$ be the partial sums of random walk with mean zero and finite variance. Angel et al. show that the LIS of the partial sum sequence $\left(S_{1}, \ldots, S_{n}\right)$ has length $n^{1 / 2+o(1)}$. They do not shed any light on what happens when the finite variance hypothesis is removed. When the second moment, and possibly the first, are undefined, it makes sense to consider other ways to keep the walk from having a drift. Here we consider random walk trajectories whose increments are symmetric about zero. We show that random walks whose increments have fat tails will have a longer LIS than do those with finite variance.

The cleanest model in which this occurs is the so-called Ultra-fat tailed distribution, which is a distribution not on $\mathbb{R}$ but on a non-archimedean totally ordered

Received by the editors February 6th, 2016; accepted March 7th, 2017.

2010 Mathematics Subject Classification. 60C05.

Key words and phrases. Ultra-fat tailed distribution, stable law, LIS, NBU.

Research supported by NSF grants \# DMS-1209117 and DMS-1612674. 
space $\mathcal{S}$ described in Section 2 below. There, we are able to show that the LIS has length at least $n^{0.69}$; see Theorem 2.1 below. However, the result also holds for real random walks with fat tails, such as the symmetric stable- $\alpha$ when $\alpha$ is sufficiently small. We also show that the LIS has length at most $n^{0.82}$. Neither of these exponents is believed to be sharp, however empirical studies suggest that the LIS exponent for the Ultra-fat tailed distribution is roughly 0.72 , so nearer to our lower bound. Numerical evidence also suggests that for stable laws, the exponent varies, interpolating between this and $1 / 2$.

The organization of the rest of the paper is as follows. The next section contains definitions, notation and preliminary facts. Section 4 proves the $n^{\beta_{0}+o(1)}$ lower bound with an explicit constant $\beta_{0}$ slightly larger than 0.69 . Section 5 proves the $n^{\beta_{1}+o(1)}$ upper bound, with an explicit constant $\beta_{1}$ slightly less than 0.9. Section 6 extends the lower bound from the Ultra-fat tail case to actual fat-tailed distributions. We conclude with some further remarks and questions.

\section{Definitions and results}

2.1. Ultra-fat tailed distribution. We begin by defining the state space $\mathcal{S}$, which is a free $\mathbb{Z}$-module with one generator $x$ for each $x \in(0,1)$. In other words, elements of $\mathcal{S}$ are finite formal linear combinations of the symbols $\{x: 0<x<1\}$ with coefficients in $\mathbb{Z}$. There should be no confusion between the formal symbol $x$ and the real number $x$ as coefficients take only integer values and are always written on the left.

Endow $\mathcal{S}$ with the lexicographic order. Formally, if $\alpha=\sum_{x \in F} a_{x} x$ and $\beta=$ $\sum_{x \in G} b_{x} x$, we may define this order relation by induction on the minimum length $m \wedge n$ of $\alpha$ and $\beta$ as follows. For $\alpha=\sum_{x \in F} a_{x} x \in \mathcal{S}$, define its degree by $|\alpha|:=$ $\sup \left\{t: a_{t} \neq 0\right\}$. By convention $|0|=0$. We define comparisons to 0 by $\alpha>0$ if and only if $|F|>0$ and $a_{|\alpha|}>0$ and $\alpha<0$ if and only if $-\alpha>0$. For elements $\alpha=\sum_{x \in F} a_{x} x$ and $\beta=\sum_{x \in G} b_{x} x$, assuming $|F|,|G|>0$, inductively define $\alpha>\beta$ if and only if one of the following conditions holds.

(i) $|\alpha|=t>|\beta|$ and $a_{t}>0$;

(ii) $|\beta|=t>|\alpha|$ and $b_{t}<0$;

(iii) $|\alpha|=t=|\beta|$ and $a_{t}>b_{t}$;

(iv) $|\alpha|=t=|\beta|$ and $a_{t}=b_{t}$ and $\alpha-a_{t} t>\beta-b_{t} t$.

This defines a total order on $\mathcal{S}$ consistent with addition: $\alpha>\beta$ and $\gamma \geq \delta$ implies $\alpha+\gamma \geq \beta+\delta$.

2.2. Ultra-fat tailed random walk. Define $F:(-1,1) \rightarrow \mathcal{S}$ by $F(0)=0$ and $F(x)=$ $\operatorname{sgn}(x)|x|$ for $x \neq 0$. Let $\left\{U_{n}: n \geq 1\right\}$ be an IID collection of real random variables uniform on $(-1,1)$. The law of $F\left(U_{1}\right)$ is called the Ultra-fat tailed distribution. Let $X_{n}:=F\left(U_{n}\right)$ and $S_{n}:=\sum_{k=1}^{n} X_{k}$, with $S_{0}:=0$. The sequence $\left\{S_{n}: n \geq 0\right\}$ is called the so-called Ultra-fat tailed random walk. A sequence $n_{1}<\cdots<n_{k}$ is an increasing subsequence if $S_{n_{i}}<S_{n_{j}}$ for all $1 \leq i<j \leq k$. The Ultra-fat tailed distribution has been used elsewhere, without a formal definition; see, e.g., Limic and Pemantle (2004). The following theorem is the main result of this note.

2.3. Main result. Let $\left\{S_{n}\right\}$ be a random walk on $\mathcal{S}$ with increments from the Ultrafat tailed distribution. Let $L(t)$ denote the length of the LIS of $\left(S_{1}, \ldots, S_{t}\right)$. We 
remark that, by convention, we have not allowed $S_{0}$ to be an element of the LIS, hence the increment $X_{1}$ will play no role.

Theorem 2.1. There are numbers $1 / 2<\gamma<\delta<1$ such that as $t \rightarrow \infty$,

$$
\mathbb{P}\left(t^{\gamma} \leq L(t) \leq t^{\delta}\right) \rightarrow 1 \text {. }
$$

In particular, one can take $\gamma=0.690$ and $\delta=0.815$.

Remark. It can be shown (see Section 7) that neither exponent is sharp.

\section{Preliminary results}

We look at the growth rate of various deterministic random functions going between the time variable for the random walk and the length variable for the corresponding LIS. Because of the proliferation of notation, we will organize by using $t$ and nearby letters when possible for quantities in the time domain and $\ell$ and nearby letters for quantities in the length domain. As usual, we use upper case letters such as $L$ and $T$ for random quantities.

The random variables $\{L(t): t \geq 1\}$ have already been introduced and follow this notational scheme. For $\ell \geq 1$ let $T(\ell):=\inf \{t: L(t) \geq \ell\}$ denote the random time that the LIS first reaches length $\ell$. Thus $L(T(\ell))=\ell$ and $T(L(t)) \leq t$.

The magnitudes of the steps are the values $\left|U_{1}\right|,\left|U_{2}\right|, \ldots$. Trivially, the order type of the first $t$ of these is uniform on all $t$ ! possible orders and independent of the sign vector, which is also uniform on $\{ \pm 1\}^{t}$. This allows for the usual conditioning identities. For example, if the variable of the greatest magnitude is $U_{\sigma}$ then the order types of $\left(\left|U_{1}\right|, \ldots,\left|U_{\sigma-1}\right|\right)$ and $\left(\left|U_{\sigma+1}\right|, \ldots,\left|U_{t}\right|\right)$ are independent and uniform. Also immediate is the following Markov property. Construct the random variables $\left\{U_{n}\right\}$ as the coordinate functions on the canonical space $\Omega:=[-1,1]^{\infty}$ with normalized Lebesgue measure. Let $\theta: \Omega \rightarrow \Omega$ be the shift $\left(U_{1}, U_{2}, U_{3}, \ldots\right) \mapsto$ $\left(U_{2}, U_{3}, \ldots\right)$. Let $\mathcal{F}_{t}:=\sigma\left(U_{1}, \ldots, U_{t}\right)$ and let $\tau$ be a stopping time with respect to the filtration $\left\{\mathcal{F}_{t}\right\}$. Then conditionally on $\mathcal{F}_{\tau}$, the sequence $\left\{X_{\tau+n}\right\}$ is distributed as the unconditional sequence $\left\{X_{n}\right\}$.

This is all pretty trivial but it allows us to state two important relationships, one sub-additive and super-additive:

$$
\begin{aligned}
L(s+t) & \leq L(s)+L(t) \circ \theta^{s} ; \\
T(\ell+m) & \geq T(\ell)+T(m) \circ \theta^{T(\ell)} .
\end{aligned}
$$

Intuitively, the first of these holds because any increasing subsequence of $\left(S_{1}, \ldots\right.$, $\left.S_{s+t}\right)$ has at most $L(s)$ entries in $[s]$ and $L(t) \circ \theta^{s}$ elements in $\{s+1, \ldots, s+t\}$. The second holds because to get an increasing subsequence of length $\ell+m$ one first needs one of length $\ell$, and must then find one of length $m$ among the remainder of the sequence. These properties do not rely on the Ultra-fat tailed distribution and hold for the LIS of any random walk.

Definition 3.1 (NBU). Say that a random variable $X$ is new better than used $(N B U)$ if for every pair of positive integers $a$ and $b$,

$$
\mathbb{P}(X \geq a+b) \leq \mathbb{P}(X \geq a) \mathbb{P}(X \geq b) .
$$

The terminology comes from reliability theory Barlow et al. (1963) and Barlow and Proschan (1965), where the inequality rewritten as $\mathbb{P}(X \geq a+b \mid X \geq a) \leq \mathbb{P}(X \geq b)$ 
says that a new light bulb has a better chance of surviving $b$ units of time, than does a light bulb that has been used for $a$ units of time.

We recall a basic property of NBU variables.

Lemma 3.2. If the random variable $X$ is $N B U$ and $\mathbb{P}(X<q)=\epsilon$, then $\mathbb{E}[X] \leq q / \epsilon$.

Proof: The NBU assumption implies that $\mathbb{P}(X \geq k q) \leq(1-\epsilon)^{k}$, so $X / q$ is stochastically dominated by a Geometric Variable of mean $1 / \epsilon-1$.

Proposition 3.3. For LIS of any random walk, each random variable $L(t)$ is $N B U$.

Proof: This follows from (3.1) and (3.2). The event $\{L(t) \geq a+b\}$ is the intersection of the events $\{T(a) \leq t\}$ and $\left\{T(b) \circ \theta^{T(a)} \leq t-T(a)\right\}$. Because $T(b) \circ \theta^{T(a)}$ is independent of $\mathcal{F}_{T(a)}$,

$$
\begin{aligned}
\mathbb{P}(L(t) \geq a+b) & =\mathbb{P}(T(a) \leq t) \cdot \mathbb{P}\left(T(b) \circ \theta^{T(a)} \leq t-T(a)\right) \\
& \leq \mathbb{P}(L(t) \geq a) \cdot \mathbb{P}(L(t) \geq b) .
\end{aligned}
$$

Proposition 3.4. Let $X$ be NBU with mean $\mu$ and let $Y$ be geometric started from zero with mean $\mu$ (that is, one less than a geometric of mean $\mu+1$ ). Denote

$$
\begin{aligned}
a_{n} & :=\mathbb{P}(X \geq n) \\
A_{n} & :=\sum_{k=n}^{\infty} a_{k} \\
g_{n} & :=\mathbb{P}(Y \geq n)=\left(\frac{\mu}{1+\mu}\right)^{n} \\
G_{n} & :=\sum_{k=n}^{\infty} g_{k}=G_{0} g_{n}
\end{aligned}
$$

where $G_{0}=1+\mu$. Then for all $n$ we have $A_{n} \leq G_{n}$.

Proof: Let $t$ be the least integer such that $a_{t}<g_{t}$. Then $t$ is at least 1 because $a_{0}=g_{0}=1$. Also $t$ is finite unless $X$ and $Y$ have the same distribution because

$$
\sum_{n=0}^{\infty} a_{n}=\sum_{n=0}^{\infty} g_{n}=1+\mu \text {. }
$$

Suppose first that $n \leq t$. Then

$$
A_{n}=1+\mu-\sum_{k=0}^{n-1} a_{k} \leq 1+\mu-\sum_{k=0}^{n-1} g_{k}=G_{n} .
$$

Now suppose that $n>t$ and assume for induction that $A_{m} \leq G_{m}$ for all $m<n$. Then using the NBU property and induction,

$$
A_{n}=\sum_{k \geq n} a_{k} \leq a_{t} \sum_{k \geq n-t} a_{k}=a_{t} A_{n-t} \leq g_{t} G_{n-t}=G_{n},
$$

completing the induction. 
Corollary 3.5. Let $X$ be $N B U$ and $Y$ be geometric started from zero with the same mean. Then for any convex function $\phi$,

$$
\mathbb{E} \phi(X) \leq \mathbb{E} \phi(Y) .
$$

Remark: An equivalent conclusion is that $X$ has the distribution of some conditional expectation of $Y$.

Proof: As before, let $a_{n}$ and $A_{n}$ be tail probabilities for $X$ and their tail sums. Letting $\Delta h(n)$ denote $h(n+1)-h(n)$, we sum by parts twice to obtain

$$
\mathbb{E} \phi(X)=\phi(0)+\sum_{n=1}^{\infty} \Delta \phi(n-1) a_{n}=\phi(0)+\Delta \phi(0) A_{1}+\sum_{n=2}^{\infty} \Delta \Delta \phi(n-2) A_{n} .
$$

Similarly,

$$
\mathbb{E} \phi(Y)=\phi(0)+\Delta \phi(0) G_{1}+\sum_{n=2}^{\infty} \Delta \Delta \phi(n-2) G_{n} .
$$

Because $\phi$ is convex, $\Delta \Delta \phi(k) \geq 0$ for $k \geq 0$. Together with $A_{k} \leq G_{k}$ for all $k$ and $A_{1}=G_{1}=\mu$, this proves the corollary.

\section{Proof of the lower bound}

In this section we prove the lower bound in Theorem 2.1. In terms of universality, this direction is the more interesting, as it shows the Ultra-fat tailed walk to be in a different LIS-universality class from mean zero finite variance walks.

For $1 \leq m<n$, let $L(m, n):=L(n-m) \circ \theta^{m}$, in other words, it is the length of the LIS of $\left(S_{m}, \ldots, S_{n}\right)$ (recall that, by convention, the LIS cannot include the initial element, $\left.S_{m}\right)$. Of course $L(m, n)$ has the same distribution as $L(n-m)$. Define $\sigma(n)$ to be the almost surely unique $k \in[n] \backslash\{1\}$ such that $\left|U_{k}\right|=\max _{2 \leq j \leq n}\left|U_{j}\right|$. In other words, $\sigma(n)$ is the time at which the random walk completed its largest magnitude step among those occuring after time 1 and before time $n$. Let $\operatorname{up}_{n}:=\left\{U_{\sigma(n)}>\right.$ $0\}$ denote the event that this greatest magnitude increment was positive. The complementary event is denoted down ${ }_{n}$. On up ${ }_{n}$, one has the inequality $S_{j}>S_{i}$ whenever $j \geq \sigma(n)>i$. Therefore, the increasing subsequences of $[n]$ are precisely the unions $A \cup B$ where $A$ is an increasing subsequence of $[\sigma(n)-1]$ and $B$ is an increasing subsequence of $[n] \backslash[\sigma(n)-1]$. It follows that $L(n)=L(\sigma(n)-1)+$ $L(\sigma(n)-1, n)$ On down ${ }_{n}$, one has $S_{j}<S_{i}$ whenever $j \geq \sigma(n)>i$, hence the increasing subsequences of $[n]$ are precisely the sets that are either an increasing subsequence of $[\sigma(n)-1]$ or of $[n] \backslash[\sigma(n)-1]$. We have therefore proved:

Proposition 4.1. The sequence of random variables $\{L(n)\}$ satisfies the recursion

$$
\begin{aligned}
L(n)= & \mathbf{1}_{\text {upp}_{n}}[L(\sigma(n)-1)+L(\sigma(n)-1, n)] \\
& +\mathbf{1}_{\text {down }_{n}} \max \{L(\sigma(n)-1), L(\sigma(n)-1, n)\} .
\end{aligned}
$$

We will prove the lower bound in Theorem 2.1 by using the recursion to obtain the following lower bound on $\mathbb{E} L(n)$.

Lemma 4.2. Let $\beta_{0}$ be the positive solution to $x+2^{-1-x}=1$, whose decimal expansion begins 0.690069 . Then $\mathbb{E} L(n) \geq n^{\beta_{0}-o(1)}$. 
Proof: Let $a_{n}=\mathbb{E} L(n)$. Note that, conditional on $\sigma(n)$, the three random variables $L(\sigma(n)-1), L(\sigma(n)-1, n)$ and $\mathbf{1}_{\mathbf{u p}_{n}}$ are all independent and distributed respectively as $L(k-1), L(n-k+1)$ and Bernoulli(1/2), where $k=\sigma(n)$. Next, use $\mathbb{E} \max \{L(k-$ 1), $L(k-1, n)\} \geq \max \{\mathbb{E} L(k-1), \mathbb{E} L(k-1, n)\}=\max \left\{a_{k-1}, a_{n-k+1}\right\}=$ $a_{\max \{k-1, n-k+1\}}$ because $\left\{a_{n}\right\}$ is nondecreasing. This gives

$$
\begin{aligned}
a_{n} & =\frac{1}{2(n-1)} \sum_{k=2}^{n}\left(a_{k-1}+a_{n-k+1}\right)+\frac{1}{2(n-1)} \sum_{k=2}^{n} \mathbb{E} \max \{L(k-1), L(n-k+1\} \\
& \geq \frac{1}{n-1} \sum_{k=1}^{n-1} a_{k}+\frac{1}{n-1} \sum_{k=\lceil n / 2\rceil}^{n-1} a_{k}\left(1-\frac{1}{2} \delta_{k, n / 2}\right) .
\end{aligned}
$$

The key observation is that for $\beta<\beta_{0}$ and sufficiently large $n$,

$$
n^{\beta} \leq \frac{1}{n-1} \sum_{k=1}^{n-1} k^{\beta}+\frac{1}{n-1} \sum_{k=\lceil n / 2\rceil}^{n-1} k^{\beta}\left(1-\frac{1}{2} \delta_{k, n / 2}\right) .
$$

Indeed, dividing (4.2) through by $n^{\beta}$, the right-hand side is a Riemann sum approximation for

$$
c_{\beta}:=\int_{0}^{1} x^{\beta} d x+\int_{1 / 2}^{1} x^{\beta} d x
$$

which evaluates to

$$
\frac{1}{\beta+1}\left(2-2^{-\beta-1}\right) \text {. }
$$

As a function of $\beta$, the quantity $c_{\beta}$ decreases as $\beta$ varies over $[0,1]$, passing through the value 1 at $\beta=\beta_{0}$. Therefore, for $\beta<\beta_{0}$, we have

$$
\frac{1}{n-1} \sum_{k=1}^{n-1} k^{\beta}+\frac{2}{n-1} \sum_{k=\lceil n / 2\rceil}^{n-1} k^{\beta}\left(1-\frac{1}{2} \delta_{k, n / 2}\right)=n^{\beta}\left[c_{\beta}-o(1)\right]>n^{\beta}
$$

provided that $n>N(\beta)$, where $N(\beta)$ is sufficiently large so that the $o(1)$ term is less than $c_{\beta}-1$.

The rest is easy. Fixing $\beta<\beta_{0}$, we may pick $C=C(\beta)$ such that $a_{n} \geq C n^{\beta}$ for all $n \leq N(\beta)$. We claim, by induction, that this is true for all $n>N(\beta)$ as well. Indeed, assuming it to be true for $n-1$, we see that the right-hand side of (4.1), which is a lower bound for $a_{n}$, is at least $C(\beta)$ times the right-hand side of (4.2). Because $n>N(\beta)$, we see from (4.2) that this is at least $C n^{\beta}$, proving the claim.

We have shown that for all $\beta<\beta_{0}$ there exists a $C$ such that $\mathbb{E} L(n) \geq C n^{\beta}$ for all $n$. This completes the proof of Lemma 4.2.

Proof of lower bound in Theorem 2.1: Fix $\gamma<\beta<\beta_{0}$. The preceding lemma gives $\mathbb{E} L(n) \geq C n^{\beta}$. By Lemma 3.2, we have $\mathbb{P}\left[L(n)<n^{\gamma}\right] \leq n^{\gamma-\beta} \rightarrow 0$.

\section{Proof of upper bound}

The proof of the upper bound in Theorem 2.1 is analogous to the proof Lemma 4.2 but in the reverse direction. It reduces to the following result.

Lemma 5.1. Let $\beta_{1}$ be the positive solution to

$$
\frac{2}{1+\beta}-\int_{0}^{1 / 2} \frac{x^{\beta}(1-x)^{\beta}}{x^{\beta}+(1-x)^{\beta}}=1,
$$


whose decimal expansion begins 0.814834 . Then $\mathbb{E} L_{n} \leq n^{\beta_{1}+o(1)}$.

Before proving this, we record the following lemma.

Lemma 5.2. Let $X_{1}$ and $X_{2}$ be independent random variables both of which are $N B U$. Let $a:=\mathbb{E} X_{1} \leq b:=\mathbb{E} X_{2}$. Then

$$
\mathbb{E}\left(X_{1} \wedge X_{2}\right) \geq \frac{a b}{a+b+1} .
$$

Proof: Let $Y_{1}$ and $Y_{2}$ be independent geometric random variables (taking values $0,1,2, \ldots)$ with respective means $a$ and $b$. Corollary 3.5 applied to $-\left(X_{1} \wedge s\right)$ shows that $\mathbb{E}\left(X_{1} \wedge s\right) \geq \mathbb{E}\left(Y_{1} \wedge s\right)$ for each fixed $s$. It follows that

$$
\mathbb{E}\left(X_{1} \wedge X_{2} \mid X_{2}\right) \geq \mathbb{E}\left(Y_{1} \wedge X_{2} \mid X_{2}\right)
$$

and hence that $\mathbb{E}\left(X_{1} \wedge X_{2}\right) \geq \mathbb{E}\left(Y_{1} \wedge X_{2}\right)$. Similar reasoning shows that $\mathbb{E}\left(Y_{1} \wedge\right.$ $\left.X_{2}\right) \geq \mathbb{E}\left(Y_{1} \wedge Y_{2}\right)$. This last quantity may be computed exactly. This is one less than the minimum of two geometrics (started from 1$)$ with respective success probabilities $1 /(a+1)$ and $1 /(b+1)$, which means a combined success probability of $(a+b+1) /(a b+a+b+1)$. One less than the mean is $a b /(a+b+1)$, proving the lemma.

Proof of Lemma 5.1: Again let $a_{n}$ denote $\mathbb{E} L(n)$. Fix $n$ and again let $\sigma=\sigma(n)$ denote the time of the largest magnitude step up to time $n$. The identity $\max \{a, b\}=$ $a+b-\min \{a, b\}$ gives

$$
L(n)=L(\sigma-1)+L(\sigma-1, n)-\mathbf{1}_{\text {down }_{n}} \min \{L(\sigma-1), L(\sigma-1, n)\} .
$$

The random variables $\mathbf{1}_{\text {down }_{n}}, L(\sigma-1)$ and $L(\sigma-1, n)$ are conditionally independent given $\sigma$. Now use Lemma 5.2 with $X=L(k)$ and $Y=L(n-k)$ where $k$ is the minimum of $\sigma-1$ and $n-\sigma+1$. This gives

$$
\mathbb{E}\left[\mathbf{1}_{\text {down }_{n}} \min \{L(\sigma)-1, L(\sigma-1, n)\}\right] \geq \frac{1-o(1)}{2} \frac{\mathbb{E} L(k) \cdot \mathbb{E} L(n-k)}{\mathbb{E}[L(k)+L(n-k)]}
$$

where the $o(1)$ term is uniform in $k$ as $n \rightarrow \infty$, coming from the ratio of $a+b+1$ and $a+b$ when $a=\mathbb{E} L(k)$ and $b=\mathbb{E} L(n-k)$. Plugging this in to (5.1) after taking expectations gives

$$
a_{n} \leq \frac{2}{n-1} \sum_{k=2}^{n} a_{k}-\frac{2}{n-1} \sum_{k=2}^{\lfloor n / 2\rfloor} \frac{1-o(1)}{2} \frac{\mathbb{E} L(k) \cdot \mathbb{E} L(n-k)}{\mathbb{E}[L(k)+L(n-k)]}
$$

Again we play the trick of replacing $a_{k}$ by $k^{\beta}$ and approximating the sum by an integral. Pulling out a factor of $n^{\beta}$, the right hand side becomes

$$
n^{\beta}\left[2 \int_{0}^{1} x^{\beta} d x-\int_{0}^{1 / 2} \frac{x^{\beta}(1-x)^{\beta}}{x^{\beta}+(1-x)^{\beta}}\right] d x+o(1) .
$$

The expression (5.3) is decreasing on $[0,1]$ and passes through the value 1 at $\beta_{1}$. Now fix $\beta>\beta_{1}$, let $N(\beta)$ be large enough so that (5.3) is less than $n^{\beta}$ for all $n \geq N(\beta)$. Choosing $C$ so that $a_{n} \leq C n^{\beta}$ for $n \leq N(\beta)$, the integral approximation then shows by induction that $a_{n} \leq C n^{\beta}$ for all $n$, finishing the proof of Lemma 5.1. Invoking Markov's inequality then ayields the upper bound in Theorem 2.1. 


\section{Real random walks}

For whose who don't accept the Ultra-fat tailed distribution as a true random walk, we include the following result.

Theorem 6.1. For any $\gamma<\beta_{0}$ there are real $\alpha, C>0$ such that the length $L(n)$ of the LIS up to time $n$ of the symmetric stable walk with index $\alpha$ has expectation at least $\mathrm{Cn}^{\gamma}$.

We begin with a lemma.

Lemma 6.2. Fix any index $\alpha \in(0,2)$. Let $\hat{\ell}(n)=\hat{\ell}_{\alpha}(n)$ denote the median of $L(n)$ for the symmetric stable walk of index $\alpha$. Then the family $\{L(n) / \hat{\ell}(n): n \geq 1\}$ is uniformly integrable. In particular,

$$
\mathbb{E}\left[\frac{L(n)}{\hat{\ell}(n)} \mathbf{1}_{A}\right] \leq h(\mathbb{P}(A))
$$

for some function $h$ with $\lim _{\varepsilon \downarrow 0} h(\varepsilon)=0$.

Proof: It suffices to show that $L(n) /(\hat{\ell}(n)+1)$ has uniform exponential tails. Subadditivity (3.1) holds for any random walk, implying for any integer $k$ the inequality $\mathbb{P}(L(n) /(\hat{\ell}(n)+1) \geq k) \leq \mathbb{P}(L(n) \geq \hat{\ell}(n)+1)^{k} \leq(1 / 2)^{k}$.

Proof of Theorem 6.1: Let $W_{n}:=\max \left\{\left|X_{k}\right|: 1 \leq k \leq n\right\}$, let $\sigma_{n}$ be the almost surely unique argmax, i.e., $W_{n}=\left|X_{\sigma_{n}}\right|$, and let $Z_{n}:=\sum_{k=1}^{n}\left|X_{k}\right|$. Let up ${ }_{n}^{\prime}$ be the event that up $_{n}$ occurs and $W_{n}>Z_{n}-W_{n}$. On up ${ }_{n}^{\prime}$, the recursion in Proposition 4.1 is satisfied at $n$.

\section{Lemma 6.3.}

$$
\frac{W_{n}}{Z_{n}} \rightarrow 1 \text { as } \alpha \downarrow 0 \text {, uniformly in } n \text {. }
$$

Consequently, $\mathbb{P}\left(\operatorname{up}_{n} \backslash\right.$ up $\left._{n}^{\prime}\right) \rightarrow 0$ as $\alpha \rightarrow 0$, uniformly in $n$.

Proof of Theorem 6.1 from Lemma 6.3: On down ${ }_{n}$, the inequality is favorable: we can still choose to use only the longer segment, hence $\mathbb{E} L(n) \geq \mathbb{E} L[\max \{\sigma(n)-$ $1, n-\sigma(n)+1\}]$. On up ${ }_{n}$ the inequality goes the wrong way, but the difference is bounded above by $(L(\sigma(n)-1)+L(\sigma(n)-1, n))\left(\mathbf{1}_{\mathbf{u p}_{n}}-\mathbf{1}_{\mathbf{u p}_{n}^{\prime}}\right)$. Taking expectations,

$$
\begin{aligned}
E L_{n} & \geq \frac{1}{n-1} \sum_{k=1}^{n-1} \mathbb{E} L_{k}+\frac{1}{n-1} \sum_{k=\lceil n / 2\rceil}^{n-1} \mathbb{E} L_{k} \\
& -\mathbb{E}(L(\sigma(n)-1)+L(\sigma(n)-1, n))\left(\mathbf{1}_{\mathbf{u p}_{n}}-\mathbf{1}_{\mathbf{u p}_{n}^{\prime}}\right) .
\end{aligned}
$$

The subtracted term $\mathbb{E}(L(\sigma(n)-1)+L(\sigma(n)-1, n))\left(\mathbf{1}_{\mathbf{u p}_{n}}-\mathbf{1}_{\mathbf{u p}_{n}^{\prime}}\right)$ is $o(\hat{\ell}(n))$ by (6.1) and Lemma 6.2. The approximation by Riemann sum and the resulting inequality then finish the proof as before.

Remark. This lemma is the only place we use specific properties of the step distribution, other than symmetry. The conclusion of the theorem will therefore hold for any symmetric distribution satisfying (6.1). There are many more extreme distributions, such as $Z=R e^{X}$ where $X$ is Cauchy and $R$ is Rademacher, for which the ratio of the greatest of $n$ picks to the sum of the magnitudes of the other $n-1$ 
goes to infinity in probability. For such distributions, the conclusion holds for all $\gamma<\beta_{0}$

Proof of Lemma 6.3: It will be convenient to construct random walk steps $\left\{X_{n}^{(\alpha)}\right\}$ simultaneously for all $n \geq 1$ and $\alpha \in(0,1)$, such that for fixed $\alpha$, the collection $\left\{X_{n}^{(\alpha)}\right\}$ is IID symmetric stable- $\alpha$. To do so, let $\mathcal{B}$ denote the Borel sets of $\mathbb{R}$ and choose a probability space $(\Omega, \mathcal{F}, \mathbb{P})$ supporting an IID sequence of Poisson point processes $N_{n}: \Omega \times \mathcal{B} \rightarrow \mathbb{Z}^{+}$with intensity $|x|^{-2}$. Mapping each point $x$ to its signed $1 / \alpha$ power $x^{(1 / \alpha)}:=\operatorname{sgn}(x) \cdot|x|^{1 / \alpha}$ produces a Poisson process of intensity $c|x|^{-1-\alpha}$. Recall (see, e.g., Durrett 2010, Chapter 3, Section 7) that the sum $\int x d N(\omega, \cdot)(x)$ of ordinates of a Poisson process with intensity $|x|^{-1-\alpha}$ is a.s. absolutely convergent for $\alpha \in(0,1)$ and has the law of a symmetric stable- $\alpha$. Therefore, the construction is completed by defining

$$
X_{n}^{(\alpha)}:=\int x^{(1 / \alpha)} d N_{n}(\omega, \cdot)(x) .
$$

Let $M_{n}$ denote the maximum magnitude of a point in the support of the random measure $\Xi_{n}:=\sum_{k=1}^{n} N_{k}$. The most that either $W_{n}^{(\alpha)}$ or $Z_{n}^{(\alpha)}$ can differ from $M_{n}^{1 / \alpha}$ is the sum of the $1 / \alpha$ powers of all other magnitudes of points in $\Xi_{n}$. Thus,

$$
\begin{aligned}
& \left|\frac{W_{n}^{(\alpha)}}{M_{n}^{1 / \alpha}}-1\right| \leq \int\left(\frac{|x|}{M_{n}}\right)^{1 / \alpha} d \Xi_{n}^{*}(x) \\
& \left|\frac{Z_{n}^{(\alpha)}}{M_{n}^{1 / \alpha}}-1\right| \leq \int\left(\frac{|x|}{M_{n}}\right)^{1 / \alpha} d \Xi_{n}^{*}(x)
\end{aligned}
$$

where $\Xi_{n}^{*}$ is $\Xi_{n}$ without the atom at $\pm M_{n}$. The point process $\Xi_{n}$ is a scaled copy of the process $\Xi_{1}$ and the same is true of $\Xi_{n}^{*}$ and $\Xi_{1}^{*}$. It follows that the law of the self-scaled point process $M_{n}^{-1} \Xi_{n}$ is the same for all $n$. Consequently, the law of the random integral on the right-hand side of (6.3) and (6.4) does not depend on $n$.

For fixed sample point $\omega$ and fixed $\alpha \in(0,1)$, the atoms $x$ of $\Xi_{n}^{*}(\omega, \cdot)$ are absolutely summable and each has maximum modulus less than $M_{n}$. Hence, each $\left(|x| / M_{n}\right)^{1 / \alpha}$ goes to zero as $\alpha \downarrow 0$. By dominated convergence, the common righthand side of (6.3) and (6.4) goes to zero. Pointwise convergence implies convergence in probability; because the law of the right-hand side of (6.3) and (6.4) is independent of $n$, the convergence is uniform in $n$. Thus, uniformly in $n$, both $Z_{n}^{(\alpha)} / M_{n}^{1 / \alpha}$ and $W_{n}^{(\alpha)} / M^{1 / \alpha}$ converge in probability to 1 , implying (6.1).

\section{Further remarks and questions}

One natural question is to prove that the exponent $\lim \log L(n) / \log n$ exists.

Another is whether we can obtain better bounds on the exponent by finding a functional form for the distribution which yield an inequality when passed through the recursion.

Neither exponent $\beta_{0}$ nor $\beta_{1}$ is sharp. The proof of

$$
\liminf \frac{\log \mathbb{E} L(n)}{\log n} \geq \beta_{0}
$$

in fact computes the correct exponent, namely $\beta_{0}$, for the length of the greedy increasing subsequence. The GIS is defined by splitting the sequence at the location 
$\sigma$ of the maximum step, and, if the step is a downward step, throwing away the smaller interval rather than the interval with the shorter LIS. The length $Z_{n}$ of the greedy increasing subsequence obeys the recursion of Proposition 4.1 but with the max taken on the inside. This seems likely to give an exponent not too far from the correct exponent, but it gives up a non-negligible amount in the recursion and cannot be sharp.

The proof of

$$
\limsup \frac{\log \mathbb{E} L(n)}{\log n} \leq \beta_{1}
$$

does not, as far as we know compute anything natural. This bound could be improved by finding the correct function $\phi(n, k)$ that computes a better lower bound on $\mathbb{E} \min \{L(k), L(n-k)\}$. Lemma 5.2 is best possible assuming only the NBU property, as the geometric random variable is the extreme case. However, we know more about $L(k)$. For example, when $j$ and $k / j$ are integers then

$$
\mathbb{P}(L(k)<\varepsilon \mathbb{E} L(k)) \leq \mathbb{P}(L(k / j)<\varepsilon \mathbb{E} L(k))^{j} .
$$

If $\mathbb{E} L(j)=j^{\beta+o(1)}$ then taking choosing $j$ so that $\varepsilon=c j^{-\beta}$ makes $\mathbb{P}(L(k / j)<$ $\varepsilon \mathbb{E} L(k))<1 / 2$ and results in

$$
\mathbb{P}(L(k)<\varepsilon \mathbb{E} L(k)) \leq 2^{-\varepsilon^{(1+o(1)) / \beta}} .
$$

The lower tails on $L(k)$ are thus expected to be very small; this ought to lead to a better lower bound on $\mathbb{E} L(k) \wedge L(n-k)$, hence a better exponent in Lemma 5.1 and in Theorem 2.1.

\section{References}

O. Angel, R. Balka and Y. Peres. Increasing subsequences of random walks. ArXiv Mathematics e-prints (2012). arXiv: 1407.2860.

R. E. Barlow, A. W. Marshall and F. Proschan. Properties of probability distributions with monotone hazard rate. Ann. Math. Statist. 34, 375-389 (1963). MR0171328.

R. E. Barlow and F. Proschan. Mathematical theory of reliability. With contributions by Larry C. Hunter. The SIAM Series in Applied Mathematics. John Wiley \& Sons, Inc., New York-London-Sydney (1965). MR0195566.

R. Durrett. Probability: theory and examples, volume 31 of Cambridge Series in Statistical and Probabilistic Mathematics. Cambridge University Press, Cambridge, fourth edition (2010). ISBN 978-0-521-76539-8. MR2722836.

V. Limic and R. Pemantle. More rigorous results on the Kauffman-Levin model of evolution. Ann. Probab. 32 (3A), 2149-2178 (2004). MR2073188.

B. F. Logan and L. A. Shepp. A variational problem for random Young tableaux. Advances in Math. 26 (2), 206-222 (1977). MR1417317.

A. M. Veršik and S. V. Kerov. Asymptotic behavior of the Plancherel measure of the symmetric group and the limit form of Young tableaux. Dokl. Akad. Nauk SSSR 233 (6), 1024-1027 (1977). MR0480398. 